\title{
Onion peel tea ameliorates obesity and affects blood parameters in a mouse model of high-fat-diet-induced obesity
}

\author{
SHOGO MATSUNAGA $^{1 *}$, KAZUO AZUMA $^{1 *}$, MAYUMI WATANABE $^{2}$, TAKESHI TSUKA $^{1}$, \\ TOMOHIRO IMAGAWA $^{1}$, TOMOHIRO OSAKI ${ }^{1}$ and YOSHIHARU OKAMOTO ${ }^{1}$ \\ ${ }^{1}$ Faculty of Agriculture, Tottori University, Tottori $680-8553 ;{ }^{2}$ Finarl Co., Ltd., Tottori 680-1167, Japan
}

Received June 24, 2013; Accepted October 24, 2013

DOI: $10.3892 /$ etm.2013.1433

\begin{abstract}
The present study examined the effects of onion peel tea (OPT) in a mouse model of high-fat-diet-induced obesity. BALB/c mice were fed a high-fat diet for three weeks, followed by a normal diet with or without OPT for 28 days. OPT suppressed the increases in body weight and level of epididymal fat tissue; it also significantly reduced the serum concentrations of total cholesterol on day 14 and those of glucose and leptin on day 28 . The results indicate that OPT has anti-obesity effects in an experimental mouse model of high-fat-diet-induced obesity.
\end{abstract}

\section{Introduction}

Obesity is an increasing global health problem, and has been associated with metabolic syndrome (MetS), diabetes, cardiovascular disease, hypertension and cancer (1). The increasing incidence of obesity suggests that this epidemic is likely to worsen in the future (2). Animal models are useful tools for evaluating the potential efficacy of compounds for the prevention and treatment of obesity. It has been demonstrated that rodents fed a high-fat diet are good models of obesity, in which the dietary environment is a major contributor (3).

It has been previously shown that certain foods are beneficial for the suppression or prevention of MetS, including tea (4). Green tea is already a popular beverage, and may be easily incorporated as part of a diet designed to attenuate or prevent the symptoms of MetS (4). Catechins, in particular, are one of the major polyphenolic compounds in tea and are beneficial for the treatment of the main MetS conditions, including obesity and type-2 diabetes, as well as cardiovascular risk factors (5). Another potentially beneficial food is

Correspondence to: Professor Yoshiharu Okamoto, Faculty of Agriculture, Tottori University, 4-101 Koyama-Minami, Tottori 680-8553, Japan

E-mail: yokamoto@muses.tottori-u.ac.jp

${ }^{*}$ Contributed equally

Key words: onion peel, tea, anti-obesity, leptin, functional food onion. Onion has the capacity to regulate lipid metabolism and suppress hyperglycemia and diabetes (6). Previous studies have attributed the anti-obesity effects of onion to quercetin, one of the flavonoids present in onion peel (7-9). Tea extracted from onion peel (onion peel tea; OPT) may thus be expected to exert beneficial effects in MetS.

In the present study, the anti-obesity effects of OPT were evaluated in mice that had been fed a high-fat diet. Furthermore, the effects of OPT on blood parameters in these mice were examined.

\section{Materials and methods}

$O P T$. The present study used freeze-dried OPT, containing $1.15 \mathrm{mg} / \mathrm{g}$ quercetin. The OPT (Sarratto tamacha) was acquired from Finarl Co., Ltd. (Tottori, Japan).

Animals and diets. Twenty BALB/c mice (male, 4 weeks old) were purchased from CLEA Japan, Inc. (Osaka, Japan). The animals were maintained under standard conditions. The use of these animals and the procedures they were subjected to were approved by the Animal Research Committee of Tottori University (Tottori, Japan). Throughout the experimental period, the mice had unrestricted access to food and water.

Study design. The mice were randomized into the control and OPT groups ( $\mathrm{n}=10$ mice per group). Following habituation, all mice were fed a high-fat diet (HFD: High Fat Diet-32; CLEA Japan, Inc.) from day -21 to day 0 . The control group was then fed a normal powdered diet (CE-2; CLEA Japan, Inc.) from day 0 to day 28 , while the OPT group was fed a normal powdered diet supplemented with $5 \%(w / w)$ OPT. The mice were weighed once every seven days from day -21 to day 28 .

Blood and epididymal fat tissue were harvested from the mice on days 14 and 28 ( $n=5$ at each time-point). Blood was collected via cardiac puncture under isoflurane inhalation anesthesia. After allowing to stand for $1 \mathrm{~h}$ at room temperature, the serum was recovered by centrifugation of the blood at $1,000 \mathrm{x} \mathrm{g}$ for $10 \mathrm{~min}$ at $4^{\circ} \mathrm{C}$. The serum samples were then stored at $-80^{\circ} \mathrm{C}$ prior to analysis. Following blood collection, the animals were immediately sacrificed by cervical dislocation, and their epididymal fat tissue was harvested and weighed. 
Table I. Effects of OPT on blood chemical parameters in a high-fat-diet-induced model of obesity.

\begin{tabular}{|c|c|c|c|c|}
\hline \multirow[b]{2}{*}{ Parameter } & \multicolumn{2}{|c|}{ Control } & \multicolumn{2}{|c|}{ OPT } \\
\hline & Day 14 & Day 28 & Day 14 & Day 28 \\
\hline Glu (mg/dl) & $172.6 \pm 17.4$ & $304.2 \pm 19.6$ & $185.6 \pm 15.2$ & $242.4 \pm 28.0^{\mathrm{a}}$ \\
\hline T-cho (mg/dl) & $87.4 \pm 10.5$ & $97.0 \pm 11.1$ & $73.4 \pm 5.6^{\mathrm{b}}$ & $99.0 \pm 13.0$ \\
\hline TG (mg/dl) & $116.0 \pm 30.2$ & $186.8 \pm 8.1$ & $79.8 \pm 9.5$ & $134.4 \pm 45.0$ \\
\hline ALT (IU/l) & $34.0 \pm 4.3$ & $35.4 \pm 9.9$ & $36.0 \pm 6.6$ & $35.6 \pm 13.1$ \\
\hline AST (IU/l) & $100.8 \pm 58.6$ & $54.6 \pm 13.8$ & $73.6 \pm 15.9$ & $50.4 \pm 11.0$ \\
\hline ALP (IU/1) & $385.2 \pm 44.4$ & $366.8 \pm 12.0$ & $412.0 \pm 99.3$ & $422.6 \pm 11.0^{\mathrm{a}}$ \\
\hline GGT (IU/1) & $6.6 \pm 2.2$ & $4.4 \pm 0.5$ & $5.4 \pm 0.5$ & $4.4 \pm 0.5$ \\
\hline $\mathrm{Alb}(\mathrm{g} / \mathrm{dl})$ & $2.1 \pm 0.1$ & $2.3 \pm 0.1$ & $2.1 \pm 0.1$ & $2.2 \pm 0.1$ \\
\hline
\end{tabular}

Data are presented as the mean \pm standard deviation; $n=5$. ${ }^{\mathrm{a}} \mathrm{P}<0.01,{ }^{\mathrm{b}} \mathrm{P}<0.05$ compared with the control group, as assessed using the Tukey-Kramer test. OPT, onion peel tea; Glu, glucose; T-cho, total cholesterol; TG, triglyceride; ALT, alanine transaminase; AST, aspartate aminotransferase; ALP, alkaline phosphatase; GGT, geranylgeranyltransferase; Alb, albumin.

Blood chemical analysis. Blood chemicals were measured using a blood chemical auto analyzer (Dry-Chem 7000; Fujifilm Inc., Tokyo, Japan). Serum triglyceride (TG), total-cholesterol (T-cho), glucose (Glu), alanine transaminase (ALT), aspartate aminotransferase (AST), alkaline phosphatase (ALP), geranylgeranyltransferase (GGT) and albumin (Alb) levels were measured.

Measurement of serum leptin concentration. A sandwich enzyme-linked immunosorbent assay kit (Morinaga Institute of Biological Science, Inc., Yokohama, Japan) was used to measure the leptin concentration, in accordance with the manufacturer's instructions.

Statistical analysis. Statistical analyses were performed using the Student's t-test or one-way analysis of variance (ANOVA) and the Tukey-Kramer test. The data are presented as the mean \pm standard deviation. $\mathrm{P}<0.05$ was considered to indicate a statistically significant difference.

\section{Results}

Effects of dietary OPT on body weight and epididymal fat tissue. In the OPT group, the intake of quercetin during the experimental period was calculated to be $5-6 \mathrm{mg} / \mathrm{kg} / \mathrm{day}$. During the experimental period, the body weights of the mice were observed to increase in the control and OPT groups (Fig. 1). However, in the OPT group, the gain in body weight was attenuated compared with that of the control group. On days 14 and 21, in particular, the body weights in the OPT group were significantly lower than those of the control group $(\mathrm{P}<0.05)$.

The mean epididymal fat tissue weights are shown in Fig. 2. On day 14 , no significant difference was identified between the epididymal fat tissue weights in the control group $(0.2 \pm 0.0 \mathrm{~g})$ and the OPT group $(0.2 \pm 0.1 \mathrm{~g})$. However, on day 28 , the mean epididymal fat tissue weight of the OPT group $(0.3 \pm 0.1 \mathrm{~g})$ was significantly lower than that of the control group $(0.5 \pm 0.0 \mathrm{~g})$ $(\mathrm{P}<0.01)$.

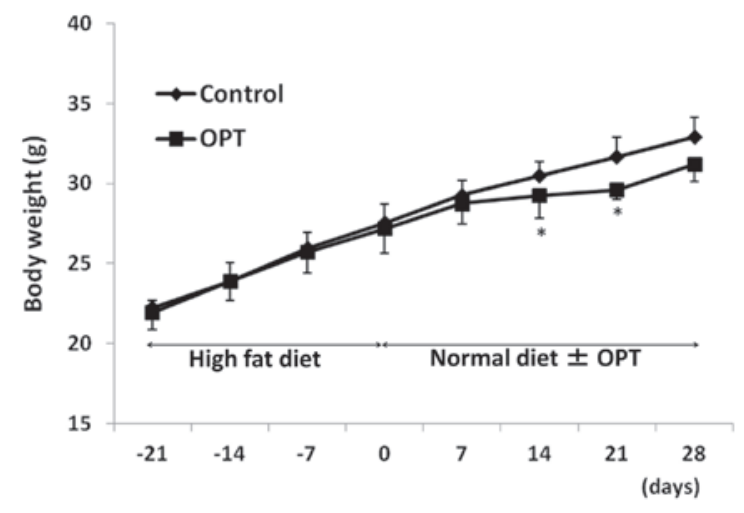

Figure 1. Effects of onion peel tea (OPT) on body weight in a high-fatdiet-induced model of obesity. The data points shown represent the means \pm standard deviation; $\mathrm{n}=5$. ${ }^{*} \mathrm{P}<0.05$ compared with the control group, as assessed using the Student's t-test.

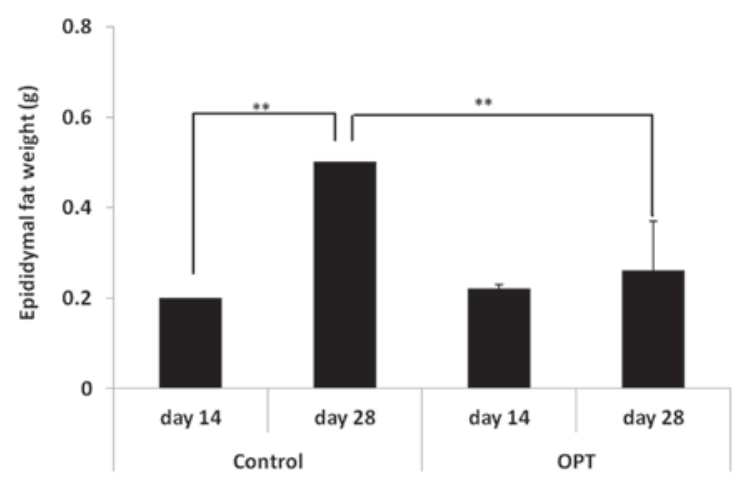

Figure 2. Effects of onion peel tea (OPT) on the weight of epididymal fat tissue in a high-fat-diet-induced model of obesity. The data shown represent the means \pm standard deviation; $\mathrm{n}=5$. ${ }^{* *} \mathrm{P}<0.01$ as determined with the Tukey-Kramer test.

Effects of OPT on blood chemical parameters. The blood chemical analysis results are shown in Table I. On day 28, the mean serum Glu concentration in the OPT group was 


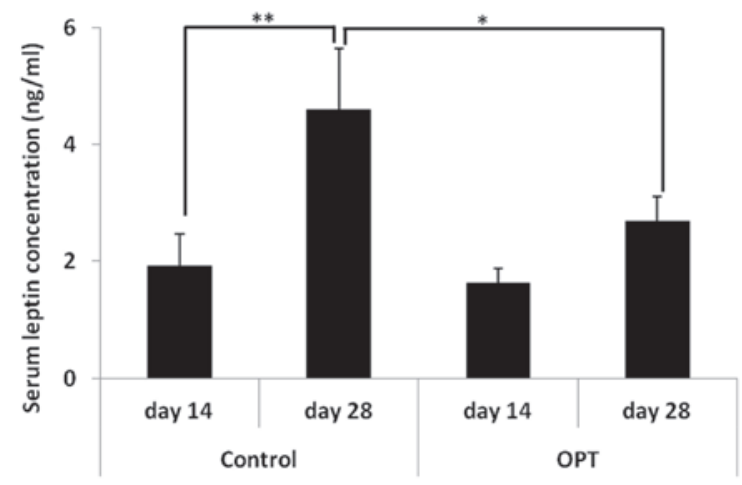

Figure 3. Effect of onion peel tea (OPT) on serum leptin concentration in a high-fat-diet-induced model of obesity. The data shown represent the means \pm standard deviation; $\mathrm{n}=5 .{ }^{*} \mathrm{P}<0.05,{ }^{* *} \mathrm{P}<0.01$ as determined with the Tukey-Kramer test.

significantly lower than that of the control group $(\mathrm{P}<0.01)$. On day 14 , however, there was no significant difference in serum Glu concentration between the groups. On day 14, the mean serum concentration of T-cho in the OPT group was significantly lower than that of the control group $(\mathrm{P}<0.05)$. On day 28 , however, there was no significant difference in mean serum T-cho concentration between the groups. On day 28, the mean serum ALP concentration in the OPT group was significantly higher than that of the control group $(\mathrm{P}<0.01)$, although there was no difference on day 14 . In the OPT group, the mean serum TG concentrations were lower than those of the control group on days 14 and 28, although these differences were not statistically significant. No statistically significant differences were identified between the groups with regard to the serum concentrations of ALT, AST, GGT or Alb on days 14 or 28 .

The serum leptin levels of the mice are shown in Fig. 3. On day 14, no significant difference was observed in the leptin level between the control group $(1.9 \pm 0.5 \mathrm{ng} / \mathrm{ml})$ and the OPT group $(1.6 \pm 0.2 \mathrm{ng} / \mathrm{ml})$. The mean serum leptin concentration in the control group was significantly higher on day 28 than that on day $14(\mathrm{P}<0.01)$. Furthermore, on day 28 , the mean serum leptin concentration in the OPT group $(2.7 \pm 0.4 \mathrm{ng} / \mathrm{ml})$ was significantly lower than that of the control group $(4.9 \pm 1.0 \mathrm{ng} / \mathrm{ml})(\mathrm{P}<0.05)$.

\section{Discussion}

In this study, dietary OPT was shown to suppress the increases in body weight and level of epididymal fat tissue in an experimental mouse model. It has been revealed that, in rodents, a high-fat diet is a major contributor to obesity (3). The results of the present study indicated that dietary OPT was capable of reducing body weight in an experimental high-fat-diet-induced model of obesity.

It has previously been suggested that dietary supplementation with onion may be beneficial for improving lipid metabolism in humans; Lee et al $(10,11)$ demonstrated that onion peel extract (OPE) was rich in quercetin and was capable of altering the expression of genes involved in cholesterol metabolism. It has also been demonstrated that OPE is able to lower blood low-density lipoprotein cholesterol and increase high-density lipoprotein cholesterol levels by increasing the expression of low-density lipoprotein receptor mRNA and mRNA encoding ATP-binding cassette transporter A1 genes (12). Moreover, a review by Sudathip et al (4) reported that green tea extract decreased blood Glu, TG and T-cho levels in diet-induced models of obesity (4). The results of the present study indicated that OPT also suppressed increases in blood Glu, TG and T-cho in a diet-induced model of obesity. Marques et al (13) demonstrated that the blood ALP levels were increased in a diet-induced mouse model of obesity. To the best of our knowledge, the correlation between obesity and an increase in blood ALP has not been completely elucidated. Further studies are required in this respect.

It has previously been indicated that the adipocytes in adipose tissue secrete a variety of proteins, known as adipocytokines, including tumor necrosis factor- $\alpha$, interleukin-6, resistin, leptin and adiponectin (14). Plasma leptin concentrations are positively correlated with adiposity (excessive body fat) and body weight changes in humans and rodents (15). Adiponectin contributes to insulin sensitivity and fatty acid oxidation, and circulating concentrations of adiponectin are inversely correlated with body mass $(16,17)$. The results of the present study indicated that OPT suppressed the secretion of leptin from adipocytes. The suppression of leptin secretion may have contributed to the reduction in body weight and the weight of epididymal fat tissue observed in the mice in the OPT group.

A high intake of plant foods (i.e. vegetables, legumes and fruits), which contain flavonoids and polyphenolic compounds, has been directly correlated with the management and prevention of obesity, type- 2 diabetes mellitus and other cardiovascular disease risk factors; among these plant foods, onions (Allium cepa) are one of the richest sources of flavonoids in the human diet (18-20). Quercetin is a major flavonol that is abundant in plant products, particularly in onions, and it has been demonstrated to possess antioxidative, anti-inflammatory and lipid-regulating properties (18,21-24). Numerous studies involving human clinical investigations, animal trials and in vitro experiments have demonstrated that phenolic substances, including quercetin, have important anti-inflammatory and anti-obesity properties $(23,25,26)$. OPT is rich in quercetin $(1.15 \mathrm{mg} / \mathrm{g})$. One possible mechanism by which OPT exerts anti-obesity effects may be via the action of quercetin. In the previous studies, the experimental animals were fed more quercetin than was taken in by the animals in the present study $(9,12)$. The results of the present study suggest that there may be another mechanism underlying the action of OPT. To enhance the understanding of any such additional mechanisms, an analysis of all of the components of OPT may be required.

In conclusion, OPT suppressed the increases in body weight and epididymal fat tissue weight normally observed with a high-fat-diet-induced model of obesity. It also significantly reduced the serum levels of T-cho and Glu. Furthermore, in the OPT group, the level of serum leptin on day 28 was significantly reduced compared with that of the control group. In combination, the results of the present study indicate that OPT may be a potent functional food for the treatment, management or prevention of obesity. 


\section{References}

1. Vucenik I and Stains JP: Obesity and cancer risk: evidence, mechanisms, and recommendations. Ann N Y Acad Sci 1271: 37-43, 2012.

2. Haslam DW and James WP: Obesity. Lancet 366: 1197-1209, 2005.

3. Bulló M, Casas-Agustench P, Amigó-Correig P, Aranceta J and Salas-Salvadó J: Inflammation, obesity and comorbidities: the role of diet. Public Health Nutr 10: 1164-1172, 2007.

4. Sudathip S, Grove KA and Lambert JD: Weight control and prevention of metabolic syndrome by green tea. Pharmacol Res 64: 146-154, 2011.

5. Thielecke $\mathrm{F}$ and Boschmann M: The potential role of green tea catechins in the prevention of the metabolic syndrome - a review. Phytochemistry 70: 11-24, 2009.

6. Corzo-Martinez M, Corzo N and Villamiel M: Biological properties of onions and garlic. Trends Food Sci Technol 18: 609-625 2007.

7. Ahn J, Lee H, Kim S, Park J and Ha T: The anti-obesity effect of quercetin is mediated by the AMPK and MAPK signaling pathways. Biochem Biophys Res Commun 373: 545-549, 2008.

8. Seiva FR, Chuffa LG, Braga CP, Amorim JP and Fernandes AA Quercetin ameliorates glucose and lipid metabolism and improves antioxidant status in postnatally monosodium glutamate-induced metabolic alterations. Food Chem Toxicol 50: 3556-3561, 2012.

9. Kim OY, Lee SM, Do H, Moon J, Lee KH, Cha YJ and Shin MJ: Influence of quercetin-rich onion peel extracts on adipokine expression in the visceral adipose tissue of rats. Phytother Res 26: 432-437, 2012.

10. Lee KH, Kim YH, Park EJ and Cho SR: Study on dietary habit and effect of onion powder supplementation on serum lipid levels in early diagnosed hyperlipidemic patients. J Korean Soc Food Sci Nutr 37: 561-570, 2008.

11. Lee KH, Park E, Lee HJ, et al: Effects of daily quercetin-rich supplementation on cardiometabolic risks in male smokers. Nutr Res Pract 5: 28-33, 2011.

12. Lee SM, Moon J, Do HJ, Chung JH, Lee KH, Cha YJ and Shin MJ: Onion peel extract increases hepatic low-density lipoprotein receptor and ATP-binding cassette transporter A1 messenger RNA expressions in Sprague-Dawley rats fed a high-fat diet. Nutr Res 32: 210-217, 2012.

13. Marques CM, Motta VF, Torres TS, Aguila MB and Mandarim-de-Lacerda CA: Beneficial effects of exercise training (treadmill) on insulin resistance and nonalcoholic fatty liver disease in high-fat fed C57BL/6 mice. Braz J Med Biol Res 43: 467-475, 2010.
14. Fasshauer M and Paschke R: Regulation of adipocytokines and insulin resistance. Diabetologia 46: 1594-1603, 2003.

15. Gnacińska M, Małgorzewicz S, Stojek M, Łysiak-Szydłowska W and Sworczak K: Role of adipokines in complications related to obesity: a review. Adv Med Sci 54: 150-157, 2009.

16. Rosen ED and Spiegelman BM: Adipocytes as regulators of energy balance and glucose homeostasis. Nature 444: 847-853, 2006.

17. Arita Y, Kihara S, Ouchi N, et al: Paradoxical decrease of an adipose-specific protein, adiponectin, in obesity. Biochem Biophys Res Commun 257: 79-83, 1999.

18. Hertog MGL, Feskens EJM, Hollman PC, Katan MB and Kromhout D: Dietary antioxidant flavonoids and risk of coronary heart disease: the Zutphen elderly study. Lancet 342: 1007-1011, 1993.

19. Knekt P, Kumpulainen J, Järvinen R, et al: Flavonoid intake and risk of chronic diseases. Am J Clin Nutr 76: 560-568, 2002.

20. Mink PJ, Scrafford CG, Barraj LM, Harnack L, Hong CP, Nettleton JA and Jacobs DR Jr: Flavonoid intake and cardiovascular disease mortality: a prospective study in postmenopausal women. Am J Clin Nutr 85: 895-909, 2007.

21. Zern TL, Wood RJ, Greene C, et al: Grape polyphenols exert a cardioprotective effect in pre- and postmenopausal women by lowering plasma lipids and reducing oxidative stress. J Nutr 135: 1911-1917, 2005.

22. Lee KH, Kim Y, Park E and Hwang HJ: Effect of onion powder supplementation on lipid metabolism in high fat-cholesterol fed SD rats. J Food Sci Nutr 13: 1371-1376, 2008.

23. Rivera L, Morón R, Sánchez M, Zarzuelo A and Galisteo M: Quercetin ameliorates metabolic syndrome and improves the inflammatory status in obese Zucker rats. Obesity (Silver Spring) 16: 2081-2087, 2008.

24. Stewart LK, Soileau JL, Ribnicky D, et al: Quercetin transiently increases energy expenditure but persistently decreases circulating markers of inflammation in C57BL/6J mice fed a high-fat diet. Metabolism 57: S39-46, 2008.

25. Hsu CL and Yen GC: Effects of flavonoids and phenolic acids on the inhibition of adipogenesis in 3T3-L1 adipocytes. J Agric Food Chem 55: 8404-8410, 2007.

26. Yen GC, Chen YC, Chang YT and Hsu CL: Effects of polyphenolic compounds on tumor necrosis factor- $\alpha$ (TNF- $\alpha)$-induced changes of adipokines and oxidative stress in 3T3-L1 adipocytes. J Agric Food Chem 59: 546-551, 2011. 